\title{
Design and implementation of VolP transceiver module based on SIP
}

\section{protocol}

\author{
Yingyan Jiang ${ }^{1,}$, Yiwen Luo ${ }^{1, b}$, Xiaodong $\mathrm{Xu}^{1, \mathrm{c}}$, Sumin Chen ${ }^{1, \mathrm{~d}}$, Peng Lin², e \\ ${ }^{1}$ Power Grid Dispatching Control Center of Guangdong Power Grid Co., Ltd, Guangdong Province, \\ China
}

${ }^{2}$ Beijing Vectinfo Technologies Co., Ltd, Beijing City, China

ajiangyingyan@gddd.csg.cn, 'luoyiwen@gddd.csg.cn, ’xuxiaodong@gddd.csg.cn, ${ }^{d}$ chensumin@gddd.csg.cn, elinpeng@vectinfo.com

\section{Key words:SIP protocol; VoIP; Voice service; message}

Abstract: SIP protocol has the advantages of simple structure, easy to expand, and so on. It has a good development prospects in network integration and business integration. Now many large business providers have begun to deploy and operate SIP based fixed network VoIP business, SIP will increasingly become the dominant voice communications. In this paper, we first introduce the architecture of VoIP system, secondly we introduce the VoIP two application layer control protocol, H.323 and sip, finally introduces the SIP messages and signaling interaction process. In order to further understand the SIP message and the signaling interaction process, this paper designs a message sending and receiving module based on SIP protocol. Java language and SIP has good compatibility, so we choose Java language, and achieve it through an interface and two classes. A single user and multiple users can send and receive a simple message ultimately.

\section{Introduction}

The application of SIP in network convergence and business integration has attracted the attention of equipment manufacturers and service providers, because the SIP protocol has the advantages of simple structure, easy to expand, and so on [1,2].

As early as 2000, Microsoft launched the instant messaging products based on the SIP protocol, and continue to optimize and upgrade, and now in the latest operating system to achieve the SIP protocol stack. Nokia and Ericsson have also developed a SIP-based end-to-end multimedia system for fixed networks and mobile networks $[3,4]$. Today, the SIP related product lines are very rich, including SIP proxy server, SIP client, SIP gateway, SIP firewall and NAT, SIP application server and so on [5,6].H.323 protocol is widely used in China's IP telephone system, the domestic network operators and equipment suppliers are actively conducting research and development and application implementation of SIP. National standards are also in full swing in the development. China Unicom, China Mobile, China Telecom, China Netcom and other large operators have launched VoIP telephone service, so that the VoIP telephone business really roots and grows in China, and toward the direction of large-scale development [7]. ZTE, Huawei and other domestic telecom equipment providers are also conducted a different degree of research and introduced the corresponding solution based on SIP-based VoIP voice business. The current market with SIP into the right track, the market share of H.323 is declining year by year, and the corresponding SIP will become more and more dominant in voice communication [8]. 


\section{VOIP Architecture}

VOIP architecture is shown in Fig. 1. The main difference between VoIP and traditional voice technology is the equipment and the network used. VoIP uses IP phones to replace traditional telephones, and adds devices that can provide digital switching, such as routers and switches, and replaces the original PSTN network with data networks.

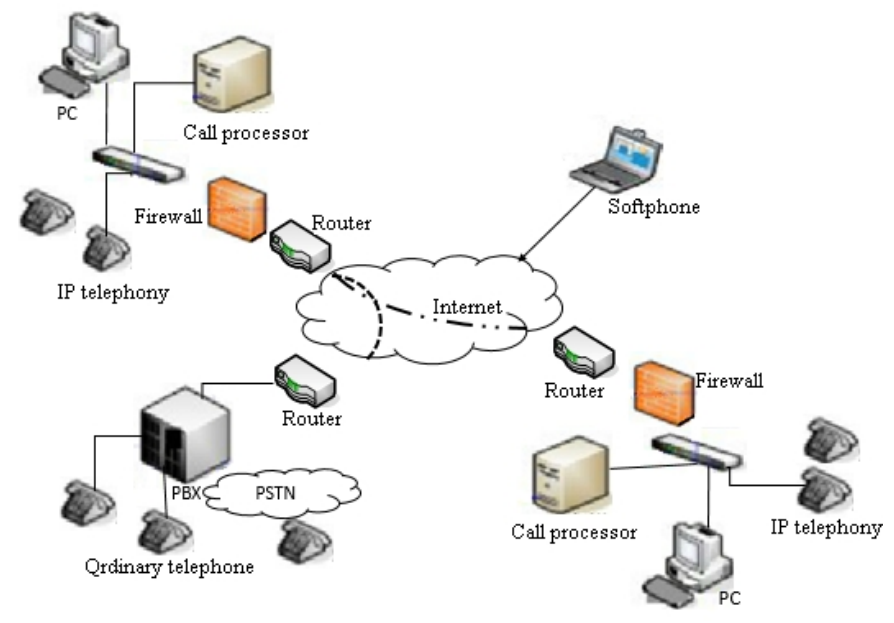

Fig. 1 VOIP Architecture

VoIP converts voice, video, data and other multimedia information into digital code, and using standard TCP/IP protocol in the IP network transmission. When establishing a voice communication service, the caller and the called party initiate the request to their respective ISP (Internet Service Provider) through the computer. Then, the ISP will confirm the identity, when successful, ISP will connect to the corresponding computer to provide data forwarding function. The official start of a call sign is that the sender uses a voice input device (such as a microphone) to transmit the voice signal to the computer, the computer for analog/digital conversion, compression, packaging after the formation of IP packets, sent to the Internet through the ISP. The Internet routes according to the destination address information in the IP packet. After the data is transmitted to the receiving end, the receiver ISP is transferred with the called terminal. Finally, the receiving computer unpacked IP packets, decompression, digital / analog conversion, and further restored to the voice information sent by a voice output device (e.g., speakers).

\section{Comparison of SIP and H.323 Protocols}

Protocol in VoIP. The main protocols involved in VoIP technology are shown in Fig. 2. Most of the organizations now use the RTP protocol originating from the IETF to carry real-time services, but the protocol for call setup and control is differentiated by the work of the standardization organizations, which is represented by H.323 and SIP.

(1) H.323 series protocol

H.323 is an umbrella proposal presented by ITU-T (ITU Telecommunication Standardization Sector). It lays the foundation for audio, video, and data communications over a VLAN or through an Internet that does not provide Quality of Service (QoS) guarantees.

The system elements of H.323 mainly consist of four parts: terminal, gateway, gatekeeper and multipoint control unit (MCU). 


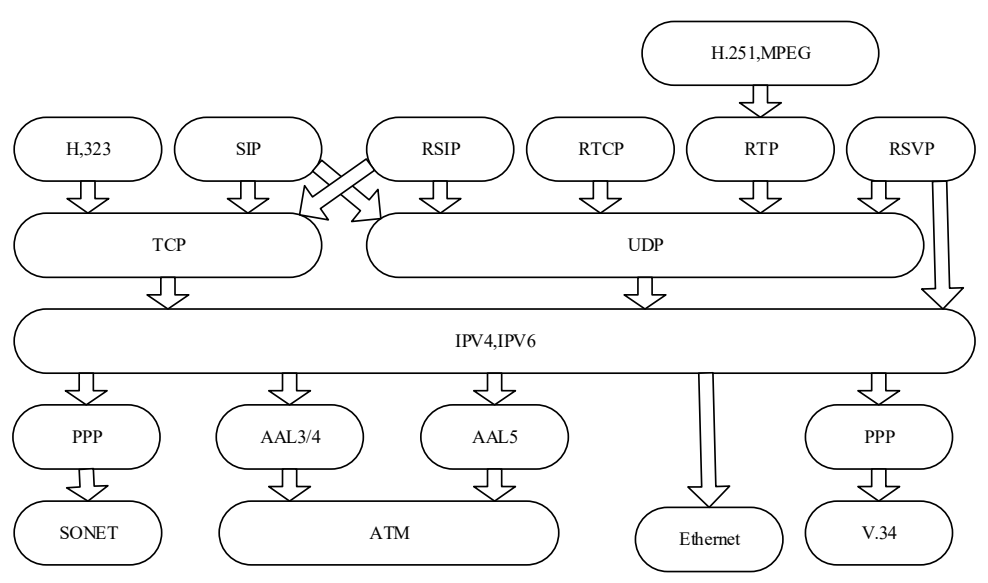

Fig. 2 Main protocols of VoIP

Terminals are often referred to as endpoints, mainly provide point-to-point and multipoint services for integrated services. The gateway is connected to the PSTN or ISDN so that the H.323 endpoint can exchange information. Gatekeeper mainly to achieve the terminal or gateway admission control and service address conversion. The MCU device allows two or more terminals or gateways to meet through an audio or video session.

(2) SIP protocol

SIP is an interactive signaling protocol developed by the IETF for initiating control, modification, and termination of a session. It applies the Peer to Peer mode, which assigns network functions such as call routing and session management for all nodes (including endpoints and network servers) of the SIP network. This is entirely different from traditional voice telephony, which relies entirely on central switches in the network. In addition, SIP fully reference HTTP and SMTP on the application layer protocol, and adopts C/S network architecture, which is easy to expand. SIP uses a separate SDP protocol to describe sessions, whose session encoding is text mode.

Comparison of SIP and H.323 Protocols. Both H.323 and SIP are protocols for supporting VoIP technology, but their usage and design are quite different. H.323 focuses on replacing circuit lines in existing Telecom grade telephone networks with IP lines. SIP is more likely to use the IP phone as an application on the Internet.

In addition, based on the next generation network (NGN), which is gradually entering people's mind, more widely adopted large-scale integrated IP products and IP gateways in the network, so that end-to-end systems can adopt IP to achieve pure IP Business Applications. At this time, IP-based SIP protocol will show great advantages. At the same time, SIP also provides good QoS support, enabling SIP to carry out high-quality transmission over the IP network.

In combination with the above analysis, the performance of the two protocols can be compared and summarized as shown in Table 1

Table 1 Comparison between SIP and H.323

\begin{tabular}{|c|c|c|}
\hline & SIP & H.323 \\
\hline Original intention of design & IP application & line switching \\
\hline Flexibility & High & Low \\
\hline Expansibility & High & low \\
\hline Call establishment implementation & Simple & Complex \\
\hline Applicability in NGN & High & Low \\
\hline
\end{tabular}

Accordingly, this topic finally uses SIP protocol for VoIP implementation, the following for SIP protocol analysis. 


\section{Design of the Message Sending and Receiving Module Based on SIP Protocol}

SIP Protocol Architecture. SIP architecture mainly contains two elements: user agents and network servers. The User Agent is divided into two parts: the User Agent Client and the User Agent Server. The client is responsible for initiating the call while the server is responsible for answering the call. According to the different logical functions, SIP network server can be divided into three categories, location server, register server and redirect server.

SIP Protocol Main Message. SIP network architecture uses $\mathrm{C} / \mathrm{S}$ and the message encoding (RFC 2068) uses HTTP/1.1. The ISO 10646 is the symbol set and the encoding is UTF-8. Depending on the function, the SIP protocol can be classified into two types: request and response messages.

The meaning of each part of the message is as follows:

CALL-ID: Multi-purpose parameters. For Invite and Registration requests, it can be used for repetitive detection. In the Register and Options requests, it is used to match the appropriate request and response.

From: Parameters that must exist in all requests and responses, contains the display name and address of the initiator, you can add optional tags, an optional request.

To: Domains that must exist, represents the intended purpose of the request. It is copied to the response, which is used primarily to specify several possible endpoints in a SIP Universal Resource Identifier (URI) (like a help desktop). A random tag is added in the response so that the client distinguishes the source of the response.

Cseq: Domains that must exist are made up of method names and unsigned sequence numbers. In a call, the requested sequence number starts with a random number, and the sequence number of the new request is increasing. Except for $\mathrm{ACK}$ and Cancel requests. The requested Cseq value must be copied by the server in the associated response.

Via: The record of the request path to ensure that the response can be forwarded along the same path.

Contact: The most useful one in the header, which provides a URL for use in future conversations on a particular session.

Max-Forwards: Used to limit the number of forwarding nodes (also called hops) that a request was sent to the destination address.

Allow: Does not establish a connection with a specific session description, but rather indicates the set of methods that are supported in the request message.

Content-Length: Eight bits of the message body.

Supported: Indicates that the functionality is supported by the server.

User-Agent: Contains the user terminal information that initiated the request.

Content-Type: The type of media that describes the content of the message body.

(1) Response message

As the start line, the status line of the SIP response message mainly contains status codes, protocol versions, and related text phrases. The response message is a response to the request message indicating the success or failure of the call. Status codes can distinguish different types of response messages. The status code is a three-digit integer, one for the response type, and two for the detailed description of the response.

(2) Response message format

The SIP message consists of two parts, the SIP response message header and a set of parameter lines, which are distinguished by newlines. The specific message header is interpreted the same as the request message. The difference is that its starting line consists of version number, status code, and descriptive phrase. 
(3) A typical SIP proxy call

The two parties send a Register request to the registration server in the respective domain respectively. If the response is $200 \mathrm{OK}$, the registration process is completed.

\section{The Message Sending and Receiving Module Design.}

To further understand the SIP message and signaling interaction process, it is decided to design a message sending and receiving module base on SIP protocol [13].

(1) Select the programming language

The choice of programming language directly determines the ease of design. After reviewing the information, it is understood that the corresponding SIP protocol stack has been introduced for SIP applications. And the SIP Servlets specification provides a series of Java API and a server development model based on container application. All these indicate that SIP and Java have good compatibility, so Java language is used for programming.

(2) Programming idea

The goal of this design is to write a control interface that can send and receive simple messages. It is decided to achieve by an interface (Message Processor) two classes (SipLayer, TextClient). The interface is the callback interface, separating the SIP layer from the GUI. The first class, SipLayer, is used to process all SIP traffic, instantiated by the TextClient class, and called back through the interface. The second class, the TextClient as the main class, includes the swing window that applies the widget in addition to instantiating the objects in the SipLayer. Because the programming of the interface is relatively simple, the content of the TextClient class is relatively routine, and the contents of the SIP protocol part are less. Therefore, here only through Fig. 6 to describe the SipLayer programming.

(3) Problems encountered and solutions

(1) Because the Java language is not familiar with, so the initial design encountered many problems. But these problems are effectively solved by looking for information. Finally, through the reference and study of some relevant examples, the expected goal is achieved.

(2) The original run of the program should be displayed for the interface, but always appears to remind to enter the user's information in the console. Troubled by this problem for a long time, and finally learned that the following instructions need to be used when running: java -jar textclient.jar $<$ username $><$ port $>$

Therefore, you need to generate buil.xml in Ant, and then packaged three files (.Classpath, .Project, build.xml) into jar files, and finally run on the command line.

(3) When running, the interface of the individual user sending and receiving information is displayed correctly. But when you want to generate multiple users, prompt error that the address has been occupied. After checking, found that two users set the same port. Because the interface is set to automatically obtain the local address, so the two user gets the same address for the same host. In this case, only different ports can be assigned to achieve multi-user establishment. 
(4) Results show

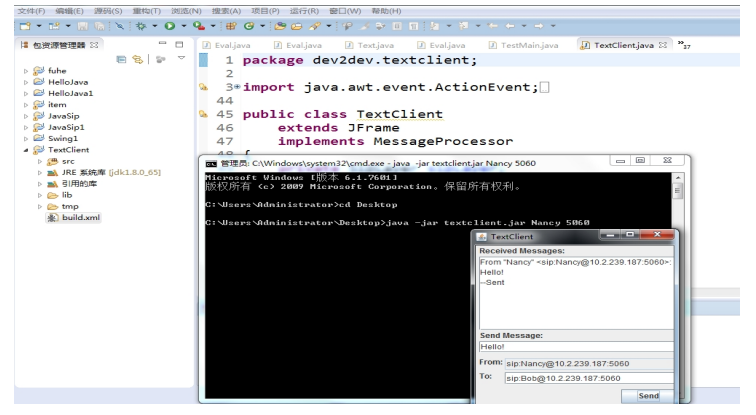

Fig. 3 The message sending and receiving interface for the single user

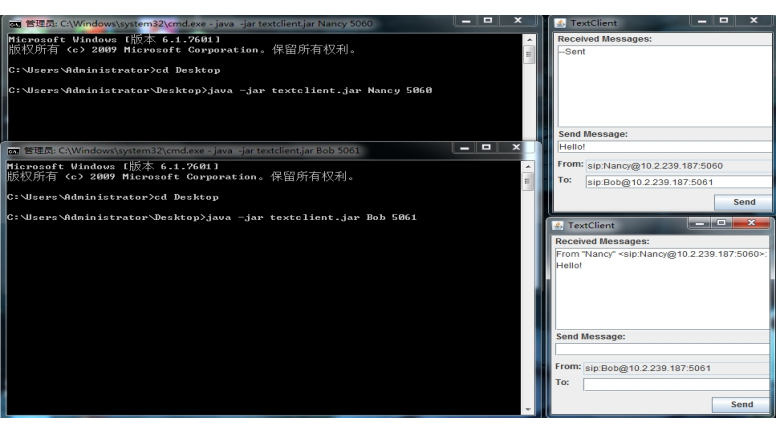

Fig. 4 The message sending and receiving interface for the multi user

\section{Conclusion}

This paper first introduces the VoIP system architecture and pays attention to the difference between it and traditional telephone. Secondly, it mainly introduces two application layer control protocols of VoIP, H.323 and SIP, and selects SIP to configure VoIP voice service after comparing the performance of the two protocols. Finally, introduce SIP messages and signaling interaction processes. In order to deepen the understanding of SIP signaling interaction, using Java language to complete the design of a message sending and receiving module based on SIP protocol, which can realize the simple message sending and receiving between single user and multi user.

\section{Acknowledgements}

This work was financially supported by Research and Application on Intelligent Operation Management Technology in Voice Exchange Network (036000KK52160009).

\section{References:}

[1]Daniel Collins, in: Carrier Grade Voice Over IP, McGraw-Hill Companies Publishing, New York (2004).

[2]Dengyin Zhang and Jingke Sun, in: VoIP Technical Analysis and System Design, Posts \& Telecom Press Publishing, Beijing (2003), In Chinese.

[3]Elliot Lewis, in: Configuring Cisco Voice over IP, China Machine Press Publishing, Beijing (2001), In Chinese.

[4]Xinyan Shen, in: Multimedia Transmission Network and VoIP System Design, Posts \& Telecom Press Publishing, Beijing (2003), In Chinese.

[5]RFC 2543: SIP: Session Initiation Protocol.

[6]Ao Zhang and Shuling Cheng, in: The FTTx Network with Three Networks, Posts \& Telecom Press Publishing, Beijing (2011), In Chinese.

[7]Ondrej Krajsa, Radim Sifta and Jakub Sedy, in: A Comparative Study of The Modern Access Networks, Progress in International Conference on Telecommunications, IEEE Publishers (2013), p. 123-126.

[8]Weiping Sun and Jiansheng Yu, in: Design and Example of FTTx and PON System Engineering, Posts \& Telecom Press Publishing, Beijing (2013), In Chinese. 